\title{
Finite element analysis of an optimized hybrid stiffened plate
}

\author{
Sándor Szirbik ${ }^{1}$, and Zoltán Virág ${ }^{2 *}$ \\ ${ }^{1}$ University of Miskolc, Institute of Applied Mechanics, H-3515 Miskolc, Hungary \\ ${ }^{2}$ University of Miskolc, Institute of Mining and Geotechnical Engineering, H-3515 Miskolc, Hungary
}

\begin{abstract}
This paper is devoted to the modal analysis and buckling of a stiffened plate with simple supported conditions within the framework of shell theory. The main objective of the finite element analysis is to investigate the natural frequencies of this stiffened structure subjected to uniaxial compression on two opposite edges of the plate. In this study, the numerical analysis is performed for such a design of the stiffed plate which has already been optimized for uniaxial compression, some design variables and the cost of welding, and the objective function to be minimized is defined as the material cost. The various Young's modulus of the base plate and the stiffeners are given thus assuming that the plate parts (the base plate and ribs) were made of different steel materials.
\end{abstract}

\section{Introduction}

Stiffened plates have many applications in various load-carrying structures, in particular in aerospace, automobile, marine structures, and civil engineering. These structures can be exposed to loads during their useful lifespan, namely, uniaxial, or biaxial compression, inplane bending and shear, lateral pressure, hydrostatic pressure, concentrated or distributed on a line, uniformly distributed static, dynamic loads, therefore need to be strengthened to increase the load-carrying capacity of plates by using stiffeners.

Structural optimization of stiffened plates has been worked out by book [1]. The stiffened welded plate used in this paper has been optimized as follows: The designed constraints of an investigated stiffened plate structure are the follows, while the objective function to be minimized is defined as the material cost [2]:

- Global buckling of the stiffened plate. The effect of initial imperfections and residual welding stresses are considered by defining buckling curves for reduced slenderness.

- Single panel buckling. This constraint eliminates the local buckling of the base plate parts between the stiffeners.

- Local and torsional buckling of stiffeners. These instability phenomena depend on the shape of stiffeners. The actual torsional buckling stress can be calculated in the function of the reduced slenderness.

- Distortion constraint. Large deflections due to weld shrinkage should be avoided.

\footnotetext{
* Corresponding author: gtbvir@uni-miskolc.hu
} 
Paper [2] has developed the minimum cost design of longitudinally stiffened plates using the strength calculation methods. These procedures can be also applied to many other problems in engineering practice $[3,4]$.

The finite element analysis is a powerful technique that is used, among other things, for the dynamic response of structures. More detailed descriptions of finite element procedures can be found in $[5,6]$. The dynamic behaviour of stiffened welded plates can be investigated by modal analysis which helps clarify the causes of resonant vibrations $[7,8]$. Numerical simulation possibilities are employing finite element methods for the modal and buckling analysis of stiffened plates to investigate the effect of initial geometric imperfection on the load-displacement response. The finite element analysis can be applied to the study of the effects of corrosion or other damage in stiffened plate structures focusing on changes in the natural frequencies and the elastic buckling strength [9]. Therefore, it is important to know that the structures are properly designed and constructed considering corrosion damage during their lifespan.

Furthermore, frequency response analysis is essential, mainly, in the case of moving machines, for instance, when examining the structural elements of the machine to know the frequencies at which the structure does not remain stable but may behave erratically so that there are investigations in different kind of industrial fields e.g., mining excavators [10, 11].

Some stiffened welded plates are often also exposed to high temperature over their lifetime. Consequently, the behaviour of these structures can be also investigated at high temperatures, where there the material properties change significantly with temperature, such as Young's modulus. This condition is easily taken into account in numerical simulations.

\section{Model geometry and loading}

The structure is made up of a base plate and some welded flat stiffeners of which number and size have already been optimized before the FE analysis. As shown in Figure 1, the lengths of the plate along the $x$-axis and $y$-axis are denoted by $L$ and $B$, respectively. Besides, the plate possesses a uniformly $t_{F}$ thickness along the $z$-axis. The displacement boundary conditions on the edges at $x=0$ and $x=L$ are given in the form

$$
w=0, \quad \partial^{2} w / \partial x^{2}=0
$$

and the boundary conditions on the edges of the base plate at $y=0$ and $y=B$ are prescribed according to

$$
w=0, \quad \partial^{2} w / \partial y^{2}=0
$$

where $w=w(x, y)$ is the displacement in the $z$ direction. This means that simple support conditions are subjected on all the edges of the base plate (SSSS).

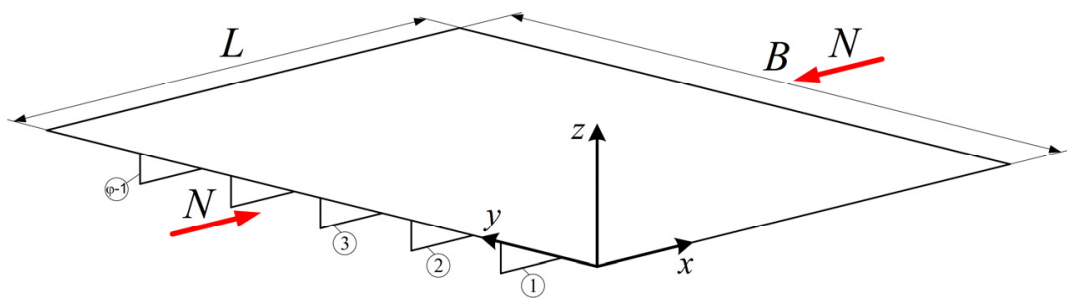

Fig. 1. Design of longitudinally stiffened plate loaded by uniaxial compression. 
Figure 1 is depicted the stiffened plate loaded by uniaxial compression on two edges of the plate through numerical simulation. The magnitude of compression force $N$ is $1.2 \times 10^{7}$ $N$ as a result of an uniformly distributed static load which can be described as boundary conditions over the length of $B$ and the stiffeners height of $h_{S}$

In numerical simulations, the parts of the structure, the steel plate and flat stiffeners are with different Young's modulus, while Poisson's ratio of $v=0.3$, density of $\rho=7.85 \times 10^{-9}$ $t / \mathrm{mm}^{3}$, and yield stress of $f_{Y}=235 \mathrm{MPa}$ remain the same. Thus, the stiffened plate is a hybrid one where the materials properties of the base plate and the stiffeners are various, which can be modelled, provided that the difference in steel quality is simply described by Young's modulus changing between $190 \mathrm{MPa}$ and $210 \mathrm{MPa}$.

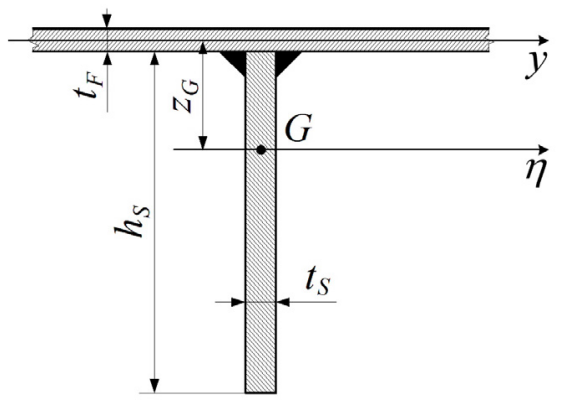

Fig. 2. Dimensions of a flat stiffener.

The optimum results for different fabrication costs calculated by Excel Solver NLP using the gradient method. The unknowns of this procedure (the thickness of the base plate $t_{F}$, the thickness of the stiffener $t_{S}$ and the number of the ribs $\left.\varphi-1\right)$ are limited in size and the welding technology is SAW - see details in Fig. 2 and paper [2]. The cost function is formulated according to the fabrication sequence. The geometrical data of the base plate are width $B=6000 \mathrm{~mm}$ and length $L=4000 \mathrm{~mm}$. First, the base plate is welded from plates of dimensions $6 m \times 1.5 \mathrm{~m}$ and $3 \mathrm{~m} \times 1.5 \mathrm{~m}$ with butt welds, then the stiffeners are welded to the base plate with fillet welds. The welding time for the first phase is given by

$$
T_{W 1}=2\left(8 \rho B L t_{F}\right)^{1 / 2}+1.3 C_{W}\left(a_{W}\right)^{n} L_{W l},
$$

in which $a_{w}(\mathrm{~mm})$ is the weld size, $L_{W l}=3 L+B$ and for SAW (submerged arc welding) butt welds for $t_{F}=4-15 \mathrm{~mm}, C_{W}\left(a_{W}\right)^{n}=0.1346 \times 10^{-3}\left(t_{F}\right)^{2}$ and for $t_{F}=15-40 \mathrm{~mm}$, $C_{W}\left(a_{W}\right)^{n}=0.1033 \times 10^{-3}\left(t_{F}\right)^{1.904}$; thus, the welding times in function of weld size $a_{w}$ for longitudinal fillet weld downhand position are known. The welding time of the second phase has the following form:

$$
T_{W 2}=3\left[\varphi\left(\rho B L t_{F}+\rho(\varphi-1) L A_{S}\right)\right]^{1 / 2}+1.3 \cdot 0.3394 \times 10^{-3}\left(a_{W}\right)^{2} L_{W 2},
$$

where $A_{S}$ is the area of a stiffener, $a_{W}=0.5 t_{S}$ but $a_{W m i n}=4 \mathrm{~mm}, L_{W 2}=2(\varphi-1) L$. The material cost can be defined as

$$
K_{M}=k_{M} \rho\left[B L t_{F}+(\varphi-1) L A_{S}\right]
$$

and the total cost is calculated as

$$
K_{M} / k_{M}=\rho\left[B L t_{F}+(\varphi-1) L A_{S}\right]+k_{F}\left(T_{W 1}+T_{W 2}\right) / k_{M} .
$$

Our goal is to give internationally usable solutions, thus we consider for material cost factor (steel) $k_{M}=0.5-1.2 \$ / \mathrm{kg}$, for fabrication including overheads $k_{F}=15-45$ $\$ /$ manhour $=0.25-0.75 \$ / \mathrm{min}$. Thus, the ratio of $k_{F} / k_{M}$ may vary in the range of $0-1.5$ 
$\mathrm{kg} / \mathrm{min}$. The value of 0 corresponds to the minimum volume design. The effect of Young's modulus changing in the base plate and the stiffeners has a minimal influence on the optimum results.

Table 1. Optimum results for the plate with flat stiffeners.

\begin{tabular}{|c|c|c|c|c|c|c|}
\hline $\begin{array}{c}\boldsymbol{k}_{\boldsymbol{F}} / \boldsymbol{k} \\
\boldsymbol{M}\end{array}$ & $\begin{array}{c}\boldsymbol{K}_{\boldsymbol{M}} / \boldsymbol{k}_{\boldsymbol{M}} \\
{[\mathbf{k g}]}\end{array}$ & $\begin{array}{c}\text { Number of the } \\
\text { stiffeners }(\boldsymbol{\varphi}-\mathbf{1})\end{array}$ & $\begin{array}{c}\boldsymbol{t}_{\boldsymbol{F}} \\
{[\mathbf{m m}]}\end{array}$ & $\begin{array}{c}\boldsymbol{t}_{\boldsymbol{S}} \\
{[\mathbf{m m}]}\end{array}$ & $\begin{array}{c}\boldsymbol{h}_{\boldsymbol{S}} \\
{[\mathbf{m m}]}\end{array}$ & $\boldsymbol{\varphi}$ \\
\hline 2 & 5060 & 5 & 15 & 13 & 182 & 6 \\
\hline 1 & 3921 & 9 & 11 & 12 & 168 & 10 \\
\hline 0 & 2172 & 28 & 5 & 10 & 140 & 29 \\
\hline
\end{tabular}

The higher production cost gives a thicker base plate with fewer stiffeners. An optimum result for the material cost for the SAW welding is shown in Table 1, in which the dimensions and the layout are given as a result of the optimization process [2].

\section{Finite element analysis}

\subsection{Finite element model}

Our investigation is based on the finite element method, which is the most popular numerical technique and can be used to numerically solve differential equations arising in engineering and modelling problems. The main concept is that the geometry of structure (the stiffened welded plate) subdivides into non-overlapping small parts with simple shaped geometry with well-defined stress displacement relationships, which is implemented in software by the construction of a mesh. Accordingly, in our modal and buckling investigation, the plate with stiffeners is divided into finite elements which are 8-node shell elements (S8R) belonging to conventional shell models, see details in $[5,6]$. These element types are formulated to work well in thin-walled structures.

In the finite element model, the base plate is subjected to a uniformly distributed load along the edges paralleled to the $y$-axis (on the edges at $x=0$ and $x=L$ ) and uniformly distributed static loads which are properly converted based on Table 1 are subjected on the corresponding edges of the stiffeners so that the loading is applied as a "shell edge load" in the software which is the commercial program Abaqus [6], as depicted in Figure 3.

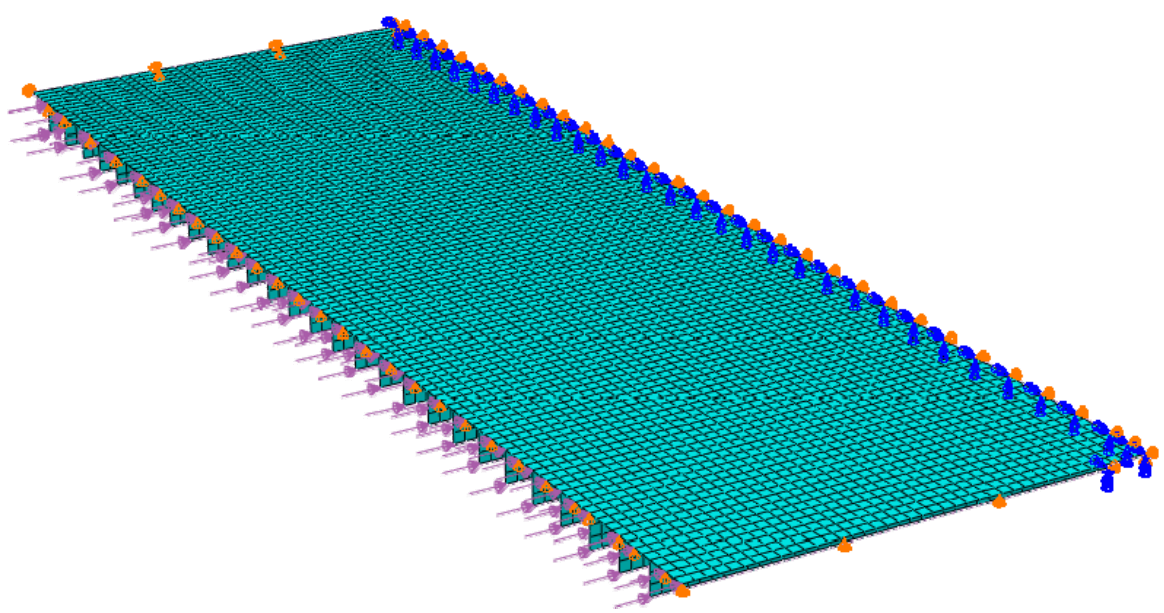

Fig. 3. Taking advantage of the symmetry, one half of the plate with boundary conditions. 
As shown in Eqs. (1) and (2), the displacement boundary condition on the four edges is given so that the base plate is simple supported. The displacement-type boundary condition in the software is applied to prescribe the corresponding displacements to the nodes of the edges.

The FE model of the optimized design of the stiffened plate contains shell elements belonging to the approximate global size is $70 \mathrm{~mm}$. Each FE model is modified by changing Young's modulus of the stiffeners, where the base plate remains original in its material properties, and vice versa. In these simulations, the eigenfrequencies of the plates with imperfection can be investigated when a compressive load is applied. Using the advantage of symmetry in geometry and loading, one half of the plate with boundary conditions is modelled, as seen in Fig. 3. The other symmetry plane, which is parallel to the $x-z$ plane, is not applied in building the FE model because it intersects the middle stiffener.

In numerical modelling, the eigenvalue extraction to calculate the natural frequencies and the corresponding mode shapes is based on the eigenvalue problem for the natural frequencies of a n-DOF undamped model. The subspace or Lanczos iteration methods described in [6] are used to perform modal frequency response analysis or to investigate the eigenvalue for buckling prediction, are applied to extract eigenvalues. Two analysis steps are used for natural frequencies belonging to the in-plane loaded plate, at the first step the applied loads and geometric nonlinearity were considered so that at the second step the load stiffness determined at the end of the first general analysis step can be included in the eigenvalue extraction.

\subsection{Numerical analysis results}

To investigate the natural frequencies, a linear perturbation analysis for the stiffened plate with flat stiffeners is performed in the commercial software Abaqus, where in each FE model is used the same conventional element type: S8R. Among the optimum results shown in Table 1 for the SAW welding the one where the number of stiffeners is 28 is investigated and presented in Table 2. In the table, the values are given in relation to Young's modulus of the base plate and the stiffener. The calculations for the other two cases in Table 1 are not reported separately here, as the same conclusions are drawn from them.

Table 2. Comparison of natural frequencies in function modulus of elasticity E [GPa].

\begin{tabular}{|c|c|c|c|c|c|}
\hline Mode No. & $\mathbf{2 1 0}-\mathbf{1 9 0}$ & $\mathbf{2 1 0}-\mathbf{2 0 0}$ & $\mathbf{2 1 0}-\mathbf{2 1 0}$ & $\mathbf{2 0 0}-\mathbf{2 1 0}$ & $\mathbf{1 9 0}-\mathbf{2 1 0}$ \\
\hline $\mathbf{1 .}$ & 80.866 & 85.156 & 89.083 & 87.041 & 84.833 \\
\hline $\mathbf{2 .}$ & 84.779 & 89.005 & 93.016 & 91.303 & 89.676 \\
\hline 3. & 92.366 & 97.493 & 102.44 & 101.81 & 101.42 \\
\hline 4. & 103.85 & 110.24 & 116.23 & 116.67 & 117.51 \\
\hline $\mathbf{5 .}$ & 116.27 & 123.48 & 130.19 & 131.31 & 132.97 \\
\hline
\end{tabular}

The first five eigenfrequencies [rad/s] belonging to the in-plane loaded plate can be seen in Table 2, in which the first row shows the choice of modulus $\mathrm{E}$ of the plate and stiffeners. By comparing these frequencies with the simulation results without load [8], the average reduction is between $30 \%$ and $40 \%$, and the corresponding mode shapes are very similar to the results in [8], as we expected.

Abaqus is capable to investigate the effect of initial geometric imperfection in our simulations. By taking the geometric imperfections allowed by designed constraints into account, the conclusion can be drawn that the imperfections do not cause a noticeable change in the eigenfrequencies. 


\section{Conclusions}

This study is devoted to an investigation of hybrid stiffened plates. The result of optimization for different manufacturing costs leads to more plates with different geometry. The eigenfrequencies show a significant decrease based on our finite element analysis if the stiffened welded plates are subjected to uniaxial compression on two edges. Furthermore, the base plate and the stiffeners are made of different steel materials, which are described with different Young's modulus while all other material properties in our simulations remain the same. It is also shown, that the eigenfrequencies show noticeable differences when the modulus of elasticity is changed. The influence of initial geometric imperfections on frequencies is less significant. Therefore, it is important that the structure is properly designed and made from proper materials. The dynamic behaviour of the stiffened plates can be further investigated by mode superposition analysis. The finite element analysis is a powerful technique that is enabled to obtain the forced response of the plates.

The described article was carried out as part of the EFOP-3.6.1-16-2016-00011 "Younger and Renewing University - Innovative Knowledge City - institutional development of the University of Miskolc aiming at intelligent specialization" project implemented in the framework of the Szechenyi 2020 program. The realization of this project is supported by the European Union, co-financed by the European Social Fund.

\section{References}

1. J. Farkas, K. Jármai, Analysis and optimum design of metal structures (Balkema Publishers, Rotterdam, Brookfield, 1997)

2. Z. Virág, K. Jármai, Optimum design of stiffened plates for static or dynamic loadings using different ribs, Struc. Eng. and Mech., 74 (2), 255-266 (2020)

3. Gy. Kovács, J. Farkas, Optimal design of a composite sandwich structure, Sci. Eng. Compos. Mater., 23 (2), 237-243 (2016)

4. A. Al-Fatlawi, K. Jármai, Gy. Kovács, Optimal design of a fiber reinforced plastic composite sandwich structure for the base plate of aircraft pallets in order to reduce weight, Polymers 13 (5), 1-35, Paper: 834 (2021)

5. K. J. Bathe, Finite Element Procedures (Prentice-Hall Inc. Englewood Cliffs, New Jersey, 1996)

6. Abaqus 6.13 online documentation (Dassault Systems, 2015)

7. A. Mukherjee, M. Mukhopadhyay, Finite element free vibration analysis of stiffened plates, Aeronaut. J. 90, 267-273 (1986)

8. Z. Virág, S. Szirbik, Finite element modal analysis of a hybrid stiffened plate, Ann. Univ. Petroşani, Mech. Eng. 21, 115-120 (2019)

9. S. Szirbik, Z. Virág, Numerical investigation of optimized stiffened plates with damaged stiffeners, Ann. Univ. Petroşani, Mech. Eng. 22, 55-62 (2020)

10. F. D. Popescu, S. M. Radu, A. Andras, I. Kertesz (Brînaș), Simulation of the frequency response of the ERC 1400 Bucket Wheel Excavator boom, during the excavation process, New Trends Prod. Eng. 2 (1), 153-167 (2019)

11. S. M. Radu, F. D. Popescu, A. Andras, I. Kertesz (Brînaș), O. B. Tomus, Simulation and modelling of the forces acting on the rotor shaft of $B W E s$, in order to improve the quality of the cutting process, Ann. Univ. Petroşani, Mech. Eng. 20, 63-72 (2018) 